\title{
Organic materials in planetary and protoplanetary systems: nature or nurture?
}

\author{
C. M. Dalle Ore ${ }^{1,2,3}$, M. Fulchignoni ${ }^{4}$, D. P. Cruikshank ${ }^{3}$, M. A. Barucci ${ }^{4}$, R. Brunetto ${ }^{5}$, H. Campins ${ }^{6}$, \\ C. de Bergh ${ }^{3}$, J. H. Debes ${ }^{7}$, E. Dotto ${ }^{8}$, J. P. Emery ${ }^{9}$, W. M. Grundy ${ }^{10}$, A. P. Jones ${ }^{5}$, V. Mennella ${ }^{11}$, \\ F. R. Orthous-Daunay ${ }^{12}$, T. Owen ${ }^{13}$, I. Pascucci ${ }^{14,15}$, Y. J. Pendleton ${ }^{16}$, N. Pinilla-Alonso ${ }^{17}$, \\ E. Quirico ${ }^{12}$, and G. Strazzulla ${ }^{18}$
}

1 Centre International d'Ateliers Scientifiques de l'Observatoire de Paris, Paris, France

2 SETI Institute, 189 Bernardo Ave., Mountain View, CA 94043, USA e-mail: Cristina.M.DalleOre@nasa.gov

3 NASA Ames Research Center, Mail Stop 245-6, Moffett Field, CA 94035, USA e-mail: Dale.P.Cruikshank@nasa.gov

${ }^{4}$ LESIA, Observatoire de Paris, 92195 Meudon Principal Cedex, France e-mail: [marcello.fulchignoni, Antonella.Barucci, Catherine.deBergh]@obspm.fr

5 Institute d'Astrophysique Spatiale (IAS), Université Paris 11 and CNRS, 91405 Orsay, France

${ }^{6}$ Physics Department, University of Central Florida, Orlando, FL 32816, USA

7 NASA Postdoctoral Program Fellow, Goddard Space Flight Center, USA

8 INAF - Osservatorio Astronomico di Roma, via Frascati 33, 00040 Monteporzio Catone (Roma), Italy e-mail: Dotto@mporzio.astro.it

9 Earth and Planetary Sciences Dept, University of Tennessee, Knoxville, TN 37919, USA e-mail: JEmery2@utk.edu

10 Lowell Observatory, 1400 W.Mars Hill Rd., Flagstaff AZ 86001, USA e-mail: W.Grundy@lowell. edu

11 INAF Osservatorio Astronomico di Capodimonte, via Moiariello, 16, 80131 Napoli, Italy

12 UJF-Grenoble 1/CNRS-INSU, Institut de Planétologie et d'Astrophysique de Grenoble (IPAG) UMR 5274, Grenoble 38041, France

13 University of Hawaii, Institute for Astronomy, USA e-mail: owen@ifa.hawaii.edu

14 Space Telescope Science Institute, Baltimore, MD 21218, USA

15 Department of Physics and Astronomy, Johns Hopkins University, Baltimore, MD 21218, USA e-mail: Pascucci@stsci.edu

16 NASA Lunar Science Institute, Mail Stop 17-1, Moffett Field, CA 94035, USA

17 Nasa Postdoctoral Program, NASA Ames Research Center, Moffett Field, CA 94035, USA e-mail: noemi .pinilla-alonso@nasa.gov

18 INAF - Osservatorio Astrofisico di Catania, via S. Sofia 78, 95123 Catania, Italy e-mail: Gianni@oact.inaf.it

Received 27 January 2011 / Accepted 6 June 2011

\section{ABSTRACT}

Aims. The objective of this work is to summarize the discussion of a workshop aimed at investigating the properties, origins, and evolution of the materials that are responsible for the red coloration of the small objects in the outer parts of the solar system. Because of limitations or inconsistencies in the observations and, until recently, the limited availability of laboratory data, there are still many questions on the subject. Our goal is to approach two of the main questions in a systematic way:

- Is coloring an original signature of materials that are presolar in origin ("nature") or stems from post-formational chemical alteration, or weathering ("nurture")?

- What is the chemical signature of the material that causes spectra to be sloped towards the red in the visible?

We examine evidence available both from the laboratory and from observations sampling different parts of the solar system and circumstellar regions (disks).

Methods. We present a compilation of brief summaries gathered during the workshop and describe the evidence towards a primordial vs. evolutionary origin for the material that reddens the small objects in the outer parts of our, as well as in other, planetary systems. We proceed by first summarizing laboratory results followed by observational data collected at various distances from the Sun. Results. While laboratory experiments show clear evidence of irradiation effects, particularly from ion bombardment, the first obstacle often resides in the ability to unequivocally identify the organic material in the observations. The lack of extended spectral data of good quality and resolution is at the base of this problem. Furthermore, that both mechanisms, weathering and presolar, act on the icy materials in a spectroscopically indistinguishable way makes our goal of defining the impact of each mechanism challenging.

Conclusions. Through a review of some of the workshop presentations and discussions, encompassing laboratory experiments as well as observational data, we infer that both "nature" and "nurture" are instrumental in the coloration of small objects in the outer parts 
of the solar system. While in the case of some observations it is clear that the organic reddening material originated before the solar nebula (i.e. presolar grains found in meteorites), for many other cases pointers are not as clear and indicate a concurrence of both processes.

Key words. planets and satellites: composition - comets: general - circumstellar matter - Kuiper belt: general - dust, extinction

\section{Introduction}

The colors of solar system bodies are often the main (or sole) source of information available for determining the composition of the surface materials, especially in the absence of spectra showing diagnostic absorption or emission features. These combined with information on the absolute reflectance (e.g., geometric albedo), the color of reflected sunlight measured over a range of wavelengths in the visible and near-infrared $(0.3-2.5 \mu \mathrm{m})$ spectral region, are usually enough to discriminate between mineral- and ice-covered surfaces. In general terms, surfaces with a high albedo and neutral color are usually ice-covered (with $\mathrm{H}_{2} \mathrm{O}$ being most common), while medium high albedo and neutral color may indicate pulverized minerals. Low albedo and a moderately red color are suggestive of mafic minerals (e.g., olivine and pyroxene), while low albedo and very red color are usually taken to indicate a surface rich in complex, macromolecular carbonaceous material similar to the kind found in primitive carbonaceous meteorites. This latter material is often referred to by the shorthand terms, kerogen-like or "organics", but it is necessary to keep in mind the rather longer but more descriptive name given in the previous sentence when using this simplification. Furthermore, we use the term "reddening" when referring to either the amount of organics or the effects of "weathering", as explained in the text. The term "weathering" is used with reference to the gradual changes to the surface of the bodies caused by irradiation.

The idea of complex organic materials constituting the coloring agent for low-albedo asteroids appears to have originated with Gradie and Veverka (1980), who suggested that “... very opaque, very red, polymer-type organic compounds, which are structurally similar to aromatic-type kerogen", mixed with montmorillonite (clay), magnetite (iron oxide), and carbon black, could match the colors of Trojan asteroids in the spectral range $0.3-1.1 \mu \mathrm{m}$. The organic material they used in their laboratory simulations was derived from coal tar by removing the soluble components and leaving the insoluble complex solid material called kerogen.

Solid organic complexes (tholins) produced synthetically by a corona or a spark discharge in a gaseous mixture of $\mathrm{N}_{2}$ and $\mathrm{CH}_{4}$ (and other molecules in some experiments) are strongly colored, and are effective when used in scattering models to reproduce the color of Titan's atmospheric aerosols (e.g., Imanaka et al. 2004; Bernard et al. 2006). Similarly, tholins are an effective coloring agent in models of solid surfaces (e.g., Hoffman et al. 1993; Dalle Ore et al. 2009; Owen et al. 2001; and Cruikshank et al. 2005).

New views of the red coloration of solid bodies in the solar system (Fig. 1) are emerging with improvements in computational methods for scattering models and new laboratory data, and with a large and homogeneous observational data set for the spectral reflectances of Saturn's satellites and rings. Clark et al. (2008) have shown that a brightness peak in the blue spectral region seen in most of Saturn's satellites can be explained as a Rayleigh scattering phenomenon caused by submicrometer particles that form a thin coating on the ice or other surface materials. For neutral-colored surfaces, these small particles may be composed of carbon, while for red-colored surfaces, the particles

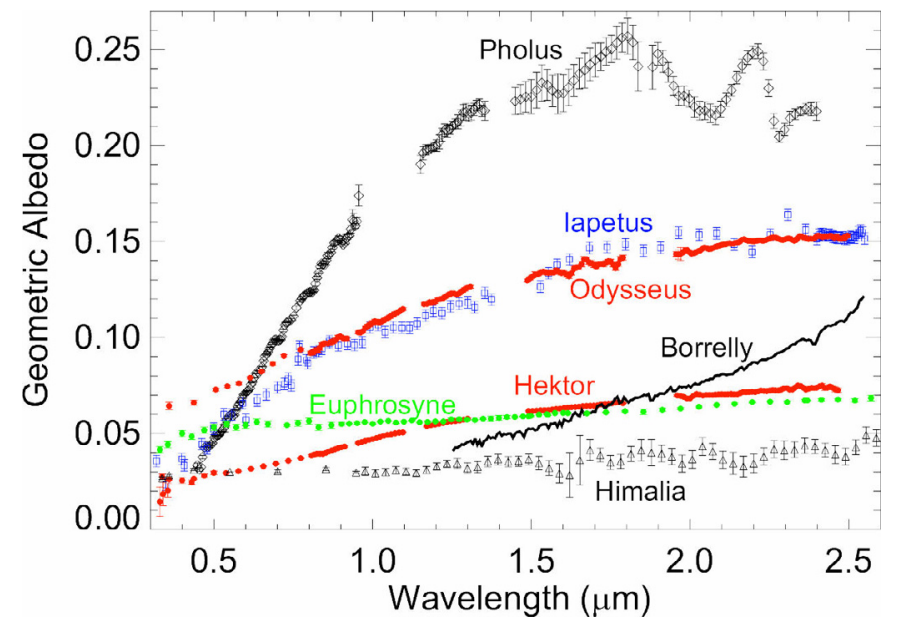

Fig. 1. Measured spectral reflectance of several solar system small bodies. Key: Pholus = Centaur object, Iapetus = Saturn Satellite, Hektor, Odysseus \& Euphrosyne $=$ Trojan asteroids, Himalia $=$ Jupiter Satellite, Borrelly = Jupiter-family comet. Figure from J. P. Emery.

may be material that is intrinsically red, such as metallic iron or iron oxides. Similarly, the regions of Saturn's rings that exhibit red color may acquire their reflectance and coloration properties from the presence of iron or iron oxides (Cuzzi et al. 2009).

With the exception of the new interpretation by Clark et al. (2008), so far, the coloration of solid bodies in the outer solar system and in debris disks of other planetary systems has been attributed to "organic" materials that can be either native to icy bodies or the products of the irradiation of volatile ices, or a combination of the two. We call the scenario of a surface being reddened by irradiation "nurture". We note, however, that both Cruikshank et al. (2001) and Emery \& Brown (2004) found that scattering models with mafic silicates can match the spectral reflectance of the red colored jovian Trojan asteroids for wavelengths shorter than $2.5 \mu \mathrm{m}$, as discussed further below. An alternative view to the "nurture" scenario is offered by Grundy (2009) where the "nurture" idea is matched with a "nature" counterpart, amply supported in recent literature (Grundy \& Stansberry 2003; Grundy 2009, and references therein). The "nature" counterpart described in detail below consists of the case in which the reddening is caused by sub-micron particles of organic material of presolar origin trapped in ice from which comes the label "nature". According to Grundy (2009) the degree of reddening varies with the concentration of the organics with respect to ice. We summarize the idea and offer laboratory and observational evidence to support the two views in an effort to unravel the puzzle that has impacted our understanding of outer solar system and planetary system chemistry. We also assess whether the evidence weighs in favor of either scenario to provide a guideline for future work and investigations. 


\section{Sub-micron organic particles and their possible influence on the colors of primitive bodies: the "nature" hypothesis}

Micron and submicron sized organic and carbonaceous particles occur in primitive meteorites, interplanetary dust particles (IDPs), and comet dust. Some could have formed in the protoplanetary nebula, via the irradiation of icy particles. Others could have survived from the cloud of gas and dust from which the Sun formed. In regions where the volatiles condensed to form ices in the outer parts of the proto-planetary nebula, these particles could have served as condensation nuclei, acquiring icy mantles, perhaps with distinct regional compositions very different from the mix of ices prevalent in the presolar cloud. Small carbonaceous particles and ices were likely the main solid ingredients from which planetesimals accreted in the outer proto-planetary nebula although comet samples retrieved by the Stardust mission also contain silicates and sulfides as significant components (Sandford et al. 2006; Sandford 2008; Clemett et al. 2010).

At visible wavelengths, the addition of colorless ice to otherwise dark organic material can dramatically increase the redness as well as the reflectance, if they are mixed at spatial scales of the order of the wavelength or less (Grundy \& Stansberry 2003; Grundy 2009). The behavior of such mixtures suggests a resolution to the dilemma posed by the inconsistent behavior of the colors and albedos of trans-neptunian objects (TNOs), Centaurs, and ecliptic comet (EC) nuclei (e.g., Jewitt 2002; Lamy \& Toth 2009). In fact, although these objects are thought to be genetically linked, much redder colors and higher albedos are found among TNOs and Centaurs than among the EC nuclei (Doressoundiram et al. 2008), culminating in consistently red colors and high albedos among dynamically cold classical belt TNOs. Cold classical TNOs, with their near circular orbits, presumably formed close to where they currently are. They may or may not be direct progenitors of Centaurs and thence ECs (Volk \& Malhotra 2008; Horner \& Lykawka 2010) but, because of their distance from the Sun, their surface compositions could offer relatively little-altered samples of the solids accreted in the outermost parts of the nebula. Radiative transfer mixing models can produce a wide variety of albedos and colors. By including sublimation loss of ice, they can also reproduce the observed color and albedo evolution as TNOs progress from the far fringes of the solar system through the giant planet-crossing Centaur zone to become ECs.

Although there is abundant evidence that icy surfaces that include carbon-bearing species are rapidly reddened and darkened as a result of radiation damage, there are also several lines of evidence to suggest that the surface colors of TNOs are not due to thin, modern radiolytic veneers, but in fact reflect their bulk compositions. Specifically, the color clusters reported by Barucci et al. (2005) are hard to explain if the surfaces of TNOs are progressively altered by radiation damage, but occasionally refreshed by impact excavation of pristine material. The colors of primary and secondary bodies among small binary TNOs are also very closely correlated (Benecchi et al. 2009). This pattern is inconsistent with time-dependent surface coloration from radiation damage, since impact erosion rates depend on object size. TNOs could be more resilient to radiation damage than previously thought, if their surface ices do not include low molecular weight organic molecules, as could happen if they mostly consist of already-highly-processed carbonaceous particles plus relatively clean $\mathrm{H}_{2} \mathrm{O}$ ice distilled by sublimation and re-condensation in the nebula.
The hollow rims of red organic globules such as seen in the Tagish Lake meteorite are also worth mentioning in this context. These shells are thought to have been produced by photolysis of the surfaces of small icy grains prior to their accretion into larger bodies (e.g., Nakamura-Messenger et al. 2006). Subsequently, the remaining ice sublimated away, leaving the less volatile organic shell. These particles tell us that at least some icy grains were already well-irradiated before they accreted into planetesimals, so we may not require the modern-day radiation environment to explain the existence of reddish organic materials in primitive bodies (although ongoing radiolysis and photolysis of icy surfaces surely does also occur).

\section{Evidence from laboratory work}

Both the "nature" and the "nurture" scenarios are likely to have played an important role in the primordial nebula before planetesimals formed. We describe some of the processes that affect carbon-rich materials and are thought to be predominant: they are mostly "nurture" mechanisms. Of the large number of different forms of carbon we consider those that are most relevant and abundant in space.

\subsection{Laboratory studies of refractory carbonaceous materials}

Carbonaceous and organic materials in the solar system and in the interstellar medium (ISM) of our own, as well as external galaxies, display a wide range of composition and structure, illustrating the rich chemistry that occurs in space. Experimental studies play a fundamental role in understanding this diversity: in the laboratory it is possible to analyze collected extraterrestrial carbon-rich materials [meteorites (see the spectrum of Orgueil in Fig. 4), micro-meteorites, IDPs, and cometary grains collected by the Stardust mission], and to synthesize, characterize, and process laboratory analogues to gain insight into the formation, origin, and evolution of the natural samples.

Among these processes UV irradiation and cosmic ion irradiation play an important role in the life-cycle of interstellar dust (Mennella et al. 2003), in the production of complex organics in the presolar nebula (Muñoz-Caro et al. 2006) as well as on the surface of TNOs and other minor bodies (Brunetto et al. 2006), and in the alteration of pre-existing carbon materials (Moroz et al. 2004; Baratta et al. 2008). Many studies point out that the early Sun was much more active than today, passing through an active $\mathrm{T}$ Tauri phase before reaching the main sequence (e.g., Feigelson \& Montmerle 1999); such strong particle and photon radiation could have had major influences on processing material during the planetary accretion phase, and more specifically on the carbon chemistry.

Several laboratories have concentrated their efforts on the production, characterization, and alteration of refractory carbonaceous materials of astrophysical interest, and in particular on the extensively studied amorphous carbons, with different degrees of hydrogenation. Different types of amorphous carbon can be produced by several techniques, such as laser pyrolysis, laser ablation or arc discharge (e.g., Scott \& Duley 1996; Mennella et al. 1999), and ion and photon irradiation of hydrocarbon-rich ices (e.g., Strazzulla \& Baratta 1991; Dartois et al. 2005). Recent studies show that the IR spectra of these materials can be used to interpret observations of the dust in the diffuse ISM of galaxies, where an important fraction of the available carbon is locked in the dust (Pendleton \& Allamandola 2002; Dartois et al. 2007; Dartois \& Muñoz-Caro 2007). Carbon soot is another interesting material (Jäger et al. 2006), due to 
its possible connection with polycyclic aromatic hydrocarbons (PAHs), and to the fact that its IR spectral features may help to interpret the nature of some of the ubiquitously observed aromatic IR bands (Pino et al. 2008). Aromatic compounds are stable molecules made of six-fold rings of carbon and hydrogen atoms (hydrocarbon). The most basic aromatic compound is the benzene ring $\left(\mathrm{C}_{6} \mathrm{H}_{6}\right)$ : PAHs are complex molecules made of fused aromatic (benzene) rings and in a terrestrial setting are a product of combustion.

Visible-IR spectra of soot and amorphous carbons differ in their chemical (e.g., hydrogen content, sp2/sp3 ratio, i.e. graphite-like to diamond-like bonding character), optical (e.g., variation of the optical gap), and structural (e.g., bond disorder, crystallites size) properties. These properties can vary according to different formation processes ("nature" scenario) but are also altered by photon and ion irradiation processes ("nurture" scenario) similar to that which is observed for irradiated organic residues. The colors of amorphous carbon vary nonuniquely as a function of the above properties, suggesting that colors might be a poor parameter to constrain the "nature" and "nurture" scenarios in carbonaceous materials.

\subsection{Ion irradiation and the colors of $C$-rich materials}

Several experiments have been performed to investigate the effects of ion irradiation on carbon-rich materials (e.g. Thompson et al. 1987). Three different kinds of materials chosen to best represent those thought to be present on the surfaces of atmosphereless outer solar system objects (i.e. ices, initially red solids, and polymer-like initially transparent solids), have been irradiated. They are: frozen $(16 \mathrm{~K})$ methane $\left(\mathrm{CH}_{4}\right)$ (Brunetto et al. 2006), asphaltite (a natural bitumen) (Moroz et al. 2004), and a film of polystyrene deposited on a silicate (olivine) (Kanuchova et al. 2010).

The irradiation of frozen $\mathrm{CH}_{4}$ shows a strong reddening and darkening of the spectra, due to the formation of an organic (C-rich) refractory residue. The color is preserved after the sublimation of the volatile species (methane and other compounds formed by irradiation) as shown by the spectrum of the residue at higher temperatures $(250 \mathrm{~K}$, Fig. 2). Further irradiation of such a solid polymer-like residue, as well as of natural red solids (e.g., asphaltite), produces a darkening and a flattening of the spectra. Recently, samples of silicates (olivine) covered with polystyrene layers have been considered. Polystyrene is considered a model for an originally transparent polymer-like material. Before irradiation, it is a transparent medium that, as ion fluence increases, exhibits spectral reddening and darkening. The reddening increases and reaches a maximum at a given fluence. Further irradiation produces further darkening, but is accompanied by a flattening of the spectrum. This process is what we refer through this paper as weathering and is considered one of the main "nurture" mechanisms.

\subsection{Laboratory evidence conclusion}

The observed reddening material could be carbonaceous in nature or could be the result of sublimation and irradiation of carbon-rich compounds (nurture). The characteristics of carbonaceous materials themselves are related to different formation processes, but can also be transformed by irradiation: their colors do not seem to correlate directly with their mode of formation, highlighting the difficulty in defining the "nature" and "nurture" scenarios. Important constraints should be sought

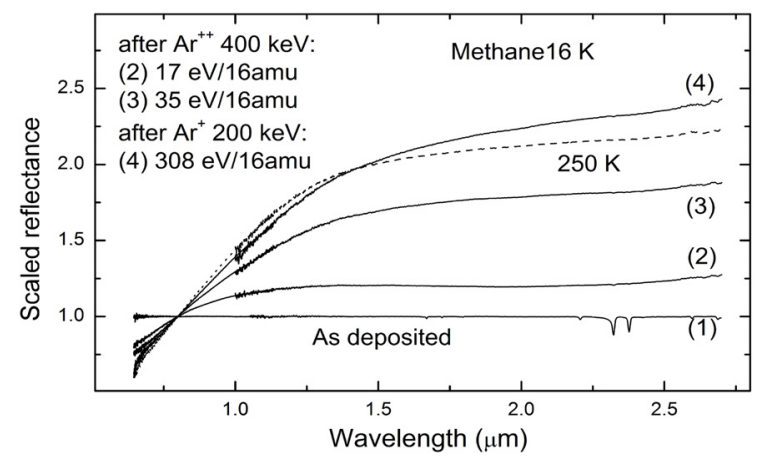

Fig. 2. Results of irradiation of $\mathrm{CH}_{4}$ ice (Brunetto et al. 2006): $\mathrm{eV} / 16$ amu units refer to the energy $(\mathrm{eV})$ adsorbed per 16 atomic mass unit. The dotted line shows the spectrum of the residue at a higher temperature $(250 \mathrm{~K})$.

in the laboratory analysis of collected extraterrestrial primitive carbons (e.g., cometary grains) to further understand the weight of one scenario over the other. On the other hand, the study of carbon-rich irradiated materials shows that weathering plays an important role in modifying the residue of $\mathrm{H}_{2} \mathrm{O}$ ice sublimation. It becomes apparent that a clear distinction between the "nature" and "nurture" scenario is very difficult when analyzing the materials that we think are responsible for most of the reddening in the solar system. Therefore, when examining the solar system evidence, we will make the simplifying assumption that the weathering process began with low-order, volatile-rich organics that are presumed to be presolar materials. We then try to infer whether "nature" or "nurture" prevails at the later stages of evolution of the materials that constitute the building blocks of planetary systems.

\section{Observational evidence outside the solar system}

\subsection{Interstellar medium (ISM)}

Fundamental to the evolution of the biogenic molecules, to the process of planetary system formation, and perhaps to the origin of life, is the connection between the organic material found in the interstellar medium and that which was incorporated in the most primitive solar system bodies. Understanding the connection between interstellar ices and organics can elucidate the inventory of materials available as the most primitive solar system bodies formed.

Star formation and the subsequent evolution of planetary systems occur in dense molecular clouds, which are comprised, in part, of interstellar dust grains gathered from the diffuse interstellar medium (DISM). Carbonaceous materials are a primary component of interstellar dust, forming in the outflow of carbon stars and the DISM. Over time, the low density DISM is swept into dense molecular clouds, the principal formation sites and repositories of most interstellar molecules. Organic compounds created in these clouds represent the first step towards the complex materials that might be responsible of the reddening in the outer solar system. In this section we examine their relationship to ices, an essential ingredient in the "nature" scenario.

\subsubsection{The nature of interstellar organics and their relationship to ices}

Infrared observational studies provide details of the solid-state features in dust grains. A series of absorption bands have been 
observed in the $3.4 \mu \mathrm{m}$ wavelength region towards bright infrared objects that are seen through high column densities of dust along multiple sightlines throughout the diffuse interstellar medium (DISM) (Pendleton et al. 1994).

Comparisons of organic residues, produced under a variety of laboratory conditions, to the diffuse interstellar medium observations have shown that aliphatic chain-like hydrocarbon grains are responsible for the spectral absorption features observed near $3.4 \mu \mathrm{m}\left(\sim 2940 \mathrm{~cm}^{-1}\right)$. The hydrocarbons in the DISM appear to carry the $-\mathrm{CH}_{2}-$ and $-\mathrm{CH}_{3}$ functional groups in the abundance ratio $\mathrm{CH}_{2} / \mathrm{CH}_{3} \sim 2.5$, and the amount of carbon tied up in this component is greater than $4 \%$ of the cosmic carbon available (Sandford et al. 1991), highlighting the importance of these materials. The Murchison carbonaceous meteorite has been shown a good analog of the interstellar observations, revealing a striking similarity between some of the hydrocarbons in the meteorite and the DISM (Pendleton \& Allamandola 2002).

Ground- and space-based telescopic observations trace interstellar organics from the diffuse to dense interstellar clouds, revealing that organic material in the DISM is predominantly hydrocarbon in nature, possessing little $\mathrm{N}$ or $\mathrm{O}$, and with the $\mathrm{C}$ distributed between aromatic ring-clusters and aliphatic forms. These data provide the first step towards identifying the nature of the reddening material in the DISM and giving us a possible link with solar system materials. The transition between the diffuse and dense cloud regions provides an interesting place to study the onset of interstellar ice chemistry. The hardy, refractory component of the DISM is expected to survive incorporation into the dense cloud environment, but to date there is no observational evidence that the DISM 3.4- $\mu \mathrm{m}$ band is present in dense molecular clouds. The deep ice band at $3.0 \mu \mathrm{m}$ detected toward many deeply embedded protostellar objects and through the quiescent dust through which background field stars are observed (Smith et al. 1993) often has a broad wing on the long-wavelength side of the band that is assumed to arise from some type of simple hydrocarbon (Sellgren et al. 1994). However, the wavelength peak of this feature and its overall structure differ appreciably from the symmetric and asymmetric aliphatic hydrocarbon bands of the DISM (Chiar \& Pendleton 2008). Mennella (2008a, 2010) proposed a scenario in which the different conditions in dense clouds with respect to diffuse regions (e.g. ice coating of the dust grains, $\mathrm{H}$ atom temperature) may inhibit the formation of $\mathrm{CH}_{2}$ and $\mathrm{CH}_{3}$ groups, while the formation of the $3.47 \mu \mathrm{m}$ band can proceed (see also Sect. 4.3).

\subsubsection{The evolution of hydrocarbonaceous dust in the ISM}

Through laboratory simulations, Dartois et al. (2004) have shown that hydrogenated amorphous carbon (denoted a-C:H) can explain not only the 3.4- $\mu \mathrm{m}$, but also the $6.85-$ and $7.25-\mu \mathrm{m}$ interstellar absorption bands if the a-C:H is hydrogen-rich ( $>50$ atomic percent $\mathrm{H}$ ). Given that recent studies of carbon dust and PAH evolution in the ISM indicate that these materials are rather susceptible to destructive processing in $\mathrm{SN}$-generated shock waves, carbonaceous solids must be rather rapidly reformed there (Serra Díaz-Cano \& Jones 2008; Micelotta et al. 2010a,b, 2011; Jones \& Nuth III 2011). Therefore it appears that a large fraction of the carbon dust in the ISM is likely to be in the form of an amorphous, re-accreted, a-C:H-like carbon, rather than in the form of crystalline graphite (pure carbon) that would be very difficult to condense in the low-density ISM. Thus, graphite is probably not an abundant component of interstellar carbon dust (e.g. Serra Díaz-Cano \& Jones 2008). For a material containing the atoms of only two elements $(\mathrm{C}$ and $\mathrm{H})$ the physical and chemical properties of a-C:H solids are surprisingly complex. Their properties can vary in response to the external conditions (e.g., temperature; photon, ion or electron irradiation). For example, a-C:H can undergo an equivalent process known as "photo-darkening", "graphitisation" or "aromatisation" when heated or exposed to UV- or ion-irradiation. This transformation leads to a closing of the optical gap and a concomitant change in the optical properties at near-IR to visible-UV wavelengths.

Dartois et al. (2004) indeed show that the thermal annealing of hydrogenated amorphous carbon materials is accompanied by an increase in the aromatic component, i.e. an "aromatization". This type of evolution for interstellar hydrocarbons was proposed previously (Duley et al. 1989; Jones 1990; Jones et al. 1990) and it was suggested to be the result of the photondriven processing of hydrogen-rich a-C:H mantles accreted onto silicate grains in the ISM. In this model the progressive loss of hydrogen, and the associated "aromatisation" of the structure was assumed to lead to materials with a lower hydrogen content, a smaller band gap, and an increased aromatic content. The end point of this evolutionary process is expected to be a low-density, aromatic-rich material that can disaggregate into its constituent aromatic molecular components (PAH-like species) with associated $\mathrm{sp} 3$ and $\mathrm{sp} 2$ carbon and hydrogen atom bridging structures (e.g., Duley 2000; Petrie et al. 2003; Pety et al. 2005; Jones 2005) as a result of UV photo-processing or fragmentation in grain-grain collisions (Jones 2005; Jones et al. 1996).

Thus, it seems that a viable source of interstellar, circumstellar or solar system "PAHs" could be provided by the natural evolution of interstellar hydrogenated amorphous carbon grains. However, in this case any "released" aromatic rich, "molecular" carriers are not the pristine and classic PAHs generally discussed in the PAH literature, but are probably more complex and contain carbon in other than purely aromatic form.

\subsection{Dense clouds}

In order to study the ice chemistry of dense clouds from the earliest stages, one needs to look at the transition zone between the diffuse and dense clouds where extinction is high enough that the grains are shielded from the harsh interstellar radiation field of the DISM allowing for ice formation. The spectroscopy of background stars seen through quiescent clouds with no star formation activity reveals that chemistry occurs before star formation begins. In fact, star formation might well begin with dust already coated with a fairly complex mixture of ices (Chiar et al. 2011). $\mathrm{NH}_{3}$-ice is an important mantle constituent in all dense cloud environments, and therefore an important reservoir of elemental $\mathrm{N}$ in these regions.

Regarding the dust component of interstellar grains, the possibility of the presence of PAH-like chemicals (aromatic hydrocarbons) in the building blocks of the solar system has been established. The next step is to understand if and how such basic primitive components could have survived the primordial nebula environment to become part of the current outer solar system bodies.

Aliphatic hydrocarbons are observed in the diffuse ISM, they are present in IDPs, cometary grains and meteorites, while they are absent in dense cloud environments. There are two possible interpretations:

1. There is no evolutionary link between the aliphatic compounds observed in the diffuse regions of the ISM and those present in solar system materials. The observation of 
organics in the two environments is a mere coincidence. In diffuse interstellar clouds, the $\mathrm{C}-\mathrm{H}$ bonds of the $\mathrm{CH}_{2}$ and $\mathrm{CH}_{3}$ groups form as a result of processing of carbon particles by $\mathrm{H}$ atoms (at thermal energies), UV photons and cosmic ions and they are destroyed when carbon grains enter into dense clouds (e.g., Mennella et al. 2002, 2003). Other processes, e.g., photoprocessing of $\mathrm{C}$ bearing ices, during solar system formation and evolution, form the aliphatic materials observed in solar system materials.

2. An evolutionary link between the aliphatic observed in diffuse ISM and solar system materials exists. In this case, a fundamental constraint to be considered is the absence of the aliphatic component in dense clouds: going from diffuse ISM to the formation site of comets/parent bodies one has to pass through the dense cloud phase where the solar system formed.

\subsection{Evolution of organics from diffuse interstellar clouds to the solar system}

The results of experiments aimed at simulating the evolution of the aliphatic carbon component in space have recently shown that an evolutionary link between the organics observed in the two environments is possible (Mennella 2008b, 2010). In fact, the same carbon grain population may absorb at $3.4 \mu \mathrm{m}$ in diffuse regions of the ISM, and at $3.47 \mu \mathrm{m}$ in dense regions. The carbon materials absorbing at $3.47 \mu \mathrm{m}$ in dense cloud/protostars, included in a comet during the formation process in the cold outer edge of the solar nebula, may thermally evolve to develop the $\mathrm{CH}_{2}$ and $\mathrm{CH}_{3}$ groups, as testified by laboratory simulations. Therefore, a unifying interpretation of how the aliphatic component appears in the main phases of its evolution in space is possible.

Studies of the interaction of hydrogen atoms at $80 \mathrm{~K}$ with carbon grains covered with a water ice layer at $12 \mathrm{~K}$ confirm that exposure of the samples to $\mathrm{H}$ atoms induces the activation of the band at $3.47 \mu \mathrm{m}$ with no evidence for the formation of aromatic and aliphatic $\mathrm{C}-\mathrm{H}$ bonds in the $\mathrm{CH}_{2}$ and $\mathrm{CH}_{3}$ functional groups. A penetration depth of $100 \mathrm{~nm}$ has been estimated for $\mathrm{H}$ atoms in the porous water ice covering carbon grains. Sample warm up to room temperature causes the activation of the IR features representing the vibrations of the $\mathrm{CH}_{2}$ and $\mathrm{CH}_{3}$ aliphatic functional groups. The presence of the $3.47 \mu \mathrm{m}$ band carrier is compatible with the evolutionary time scale limit imposed by fast cycling of materials between dense and diffuse regions of the interstellar medium. In diffuse regions the formation of the $\mathrm{CH}_{2}$ and $\mathrm{CH}_{3}$ aliphatic bands, inhibited in dense regions, takes place, masking the $3.47 \mu \mathrm{m}$ band. The activation of the $\mathrm{CH}_{2}$ and $\mathrm{CH}_{3}$ aliphatic vibrational modes at the end of $\mathrm{H}$ processing after sample warm up represents the first experimental evidence supporting an evolutionary connection between the interstellar carbon grain population, which is responsible for the $3.4 \mu \mathrm{m}$ band (diffuse regions) and contributes to the absorption at $3.47 \mu \mathrm{m}$ (dense regions), and the organics observed in interplanetary dust particles and cometary Stardust grains.

\subsection{The chemistry of protoplanetary disks and the effect of stellar UV irradiation}

Interstellar clouds evolve into proto-planetary disks and then protostars. Observations of proto-planetary disks can be used to monitor the chemical variations during the early phases of planetary evolution and to infer the chemistry in the primordial solar system. The chemical transformation of complex organic molecules during accretion and disk evolution and the level of complexity that they achieve as the protoplanetary disk enters the planet-formation stage are current research topics being pushed forward by recent dramatic advances in infrared and millimeter astronomy.

Recent observations point to chemical evolution during the first $30 \mathrm{Myr}$ of the disk lifetime. Due to the very high temperatures necessary for star formation, all materials, unless far enough from the protostar, are in gaseous form. During the condensation, planet accretion and formation phases they return to be seen in solid form. Optically thick gas-rich disks around young solar analogs are found to have rich infrared spectra indicating the presence of water, $\mathrm{OH}$, and organic molecules such as $\mathrm{C}_{2} \mathrm{H}_{2}$ and $\mathrm{HCN}$ in the warm $(\sim 700 \mathrm{~K})$ disk atmosphere within a few AU from the star (Carr \& Najita 2008; Pontoppidan et al. 2010). The large abundances of these molecules suggest that they form not only from evaporation of inward migrating ices but also from additional chemistry in the gas phase. Atomic and ionic species such as ionized $\mathrm{Ne}$ are also frequently detected (Pascucci et al. 2007; Güdel et al. 2010) and trace the very hot disk surface (several thousands $\mathrm{K}$ gas) likely photoevaporating from the star-disk system (Pascucci \& Sterzik 2009). CN, HCN, $\mathrm{H}_{2} \mathrm{CO}$ and some deuterated species are also detected at large disk radii (beyond $100 \mathrm{AU}$ ) via interferometric millimeter observations (Öberg et al. 2010).

As the inner part of the disk clears out, the observed infrared spectra dramatically change. No water molecules and no organics are detected, just atomic ionic species and the very high $\mathbf{J}$ transition of $\mathrm{OH}$, pointing to dissociation of water by the now stronger UV field that is no longer shielded by the dust (Najita et al. 2010). The outer disk does not seem to be affected by these changes: the molecular complexity observed at this stage is the same as that of optically thick gas-rich disks.

Finally, as the disk clears out most of its primordial dust (and likely gas), for systems older than $10 \mathrm{Myr}$, infrared and millimeter spectra are strikingly lacking a number of molecules, atomic and ionic species (Pascucci et al. 2006) such as $\mathrm{H}_{2}$ IR gas lines, forbidden atomic lines or $\mathrm{CO} \mathrm{mm}$ lines, that are instead observed in younger disks. This fact is attributed to a decrease of gas in the disk. According to Pascucci et al. (2006) there is less than 0.1 Jupiter mass of gas in disks that are 10 Myr old or older, implying that giant planet formation should occur in less than $10 \mathrm{Myr}$, in agreement with current giant planet formation theories.

Along with time evolution, we also have evidence for the effect of different UV fields from the different types of molecular species detected toward stars of different luminosities. While stars that are a few times more massive than the Sun with stronger UV lack almost any molecular detections in their spectra (Fedele et al. 2011; Pontoppidan et al. 2010), disks around very low-mass stars present a rich chemistry (Pascucci et al. 2009). What remains to be seen is how much of the chemistry in the disk mid-plane is impacted by the different radiation fields and what are the effects on the composition of forming planets.

\subsection{The evidence for organics in dusty disks}

Once the gas disperses in a protoplanetary disk, gas phase chemistry ceases and rocky bodies and dust are all that remain in a young planetary system. Often the collision or evaporation of planetesimals form second generation dusty debris disks. In these systems, the dust provides a unique remote laboratory to study the composition and thermal processing in the disk 
(see Apai et al. 2010, for a recent review). Both scattered light and thermal emission from these disks can provide compositional information, on the processing of organic compounds. Unfortunately, many debris disks are cold and have few spectral features in the mid-IR. If they are spatially resolved in scattered light, they are often imaged at one or two wavelengths. Therefore, because of the infancy of such observations, more questions than answers remain. The most detailed scattered light analysis of a disk comes from observations of the HR 4796A debris disk observed in seven wavelength bands that span the visible to the near-IR. From the analysis of Debes et al. (2008), a mixture of silicates and tholins were found to be the bestfitting explanation for the red character of the disk, assuming a purely Mie scattering model of solid spheres (See Fig. 3). Porous aggregates of carbon, water ice, and silicates can also fit the scattered light and IR spectral energy distribution (SED) of HR 4796A, providing an alternate explanation (Köhler et al. 2008). However, there are problems with this interpretation, including whether water ice should survive in the UV environment of an A star (Grigorieva et al. 2007).

Furthermore, neither pure tholins nor porous aggregates adequately fit the observed phase function of HR 4796A's ring in the visible (Debes et al., in prep.). Analysis of the HR 4796A ring surface brightness in the visible as a function of scattering angle for the north-east and south-west lobes of the disk show a remarkably flat inferred phase function for the constituent dust. Figure 3 shows that the data are poorly fit by either a HenyeyGreenstein phase function (as assumed by Köhler et al. 2008) or Mie scattering phase functions of pure micron-sized tholin grains (as assumed by Debes et al. 2008). Further study is required to determine the composition of the dust in the HR 4796A disk and how representative it is compared to other debris disk systems. More information on the "nature" versus "nurture" problem at the late stages of planet formation may come as more debris disks are observed in scattered light in the visible and near-IR.

\section{Observational evidence in the solar system}

Having laid the foundations of the investigation by studying the building blocks of the solar system, we proceed to examine the observational evidence at increasing distance from the Sun. We first search for evidence of organic signatures and try to quantify the organic matter content in the interplanetary material as well as meteoritic samples analyzed in the laboratory. We follow up on this with a systematic search for signs of organics in the spectra and photometric colors of small bodies (i.e. asteroids, satellites, TNOs, comets) from the asteroids in the Main Belt to the region beyond Neptune. Lastly, we analyze the behavior of colors in the outer solar system looking for correlations with the quantity of ice. This scheme is based on the fact that, according to our current knowledge of the solar nebula formation and evolution, the quantity of $\mathrm{H}_{2} \mathrm{O}$ ice increases with decreasing temperature and increasing distance from the Sun.

\subsection{Structural and chemical characterization of organic matter in meteoritic chondrites and interplanetary dust}

In order to identify the source of reddening in meteoritic chondrites and interplanetary dust particles (IDPs) we separate the organic matter into its main components.

Insoluble organic matter (IOM) is the predominant carbon material in chondrite meteorites, stratospheric IDPs and antarctic micrometeorites (AMMs). The given environment and the
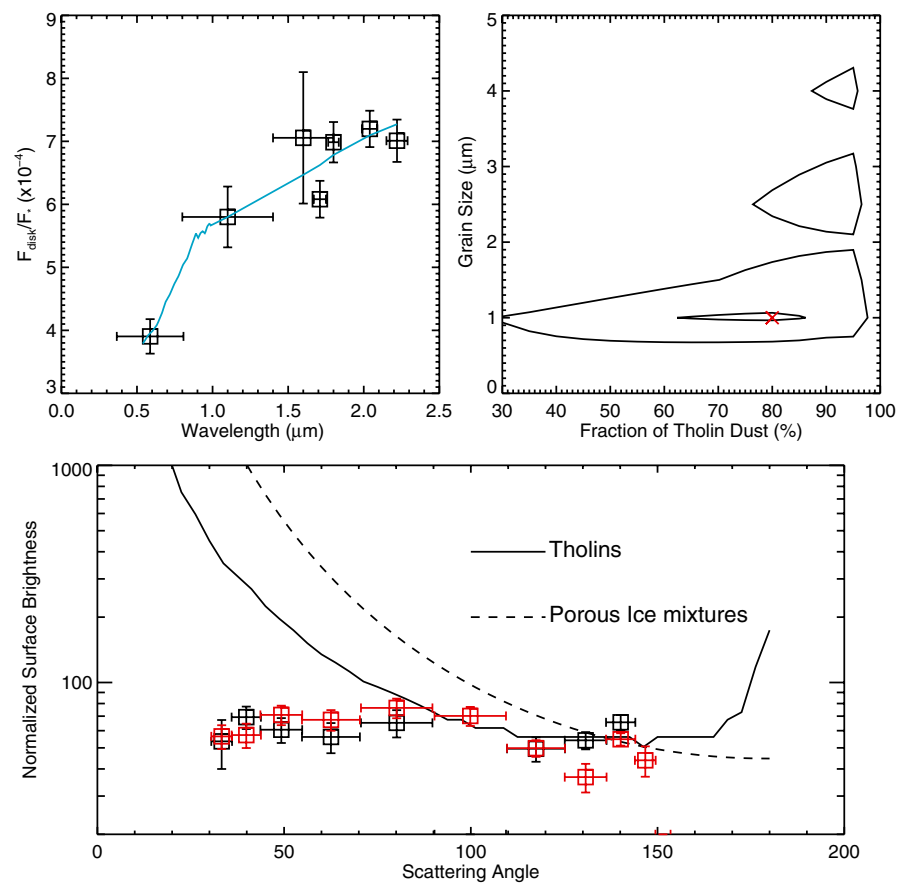

Fig. 3. (Top left) Scattering efficiency $F_{\text {disk }} / F_{\star}$ as a function of wavelength for the HR 4796A debris disk. Horizontal error bars represent the width of the filter for each image of the disk, and the solid line is the best fitting mixture of Titan tholins and astronomical silicates $\left(a_{\min }=1 \mu \mathrm{m}\right.$ (grain size), 20\% Silicates, $80 \%$ Tholins). (Top right) Contours of the $68.5 \%$ and $98 \%$ confidence levels for the models of HR 4796A's scattered light as a function of minimum grainsize and Tholin fraction. The $\mathrm{X}$ represents the best fitting model with a $\chi_{v}^{2}=0.98$. (Bottom) Relative scattered light surface brightness at visible wavelengths for the HR 4796A debris disk as a function of inferred scattering angle for dust grains in the disk. Grey squares represent the south-west lobe of the ring corrected for the brightness asymmetry present in the ansae of the disk (Schneider et al. 2009) while black squares represent the north-east lobe. Overplotted are phase functions of a) a porous mixture of ices, silicates, and carbonaceous material assuming a Henyey-Greenstein approximation to the phase function and $\mathbf{b}$ ) Tholins assuming a Mie scattering model phase function.

chemical routes to its formation have so far not been clearly elucidated. The $\mathrm{D}$ and ${ }^{15} \mathrm{~N}$ enrichments found in these materials are consistent with synthesis in a cold medium through ion-molecule reactions, but the context where these enrichments took place (molecular cloud, proto-planetary disk) is unknown (e.g., Alexander et al. 2007). The origin of the soluble organic matter (SOM) - i.e. molecules released with organic solvents or water - has been attributed to the effects of hydrothermal processes in the parent body (Alexander et al. 1998; Yabuta et al. 2005), in which SOM could be the hydrolysis product of IOM. An alternative view considers SOM as a component of the ices that were accreted along with refractory phases, and therefore with no connection with IOM (Remusat et al. 2005).

Among and within chondrites, the IOM presents a wide range of isotopic and elemental compositions, as well as large chemical and structural variations (Alexander et al. 2007; Quirico et al. 2003). These variations are controlled by heterogeneities in the precursors accreted by the parent bodies and by the effects of post-accretion processes (Cody \& Alexander 2005; Quirico et al. 2009). Post-accretion processes include low-temperature hydrothermalism (Cody \& Alexander 2005; Yabuta et al. 2005; Orthous-Daunay et al. 2010a), long-duration radiogenic thermal metamorphism (Bonal et al. 2006a, 2007; 
Busemann et al. 2007), and short-duration thermal metamorphism (Yabuta et al. 2010). IOM in interplanetary dust particules (IDPs) and antarctic micrometeorites (AMMs) has a fairly, but not strictly, similar polyaromatic structure to that of chondrites (Quirico et al. 2005; Bonal et al. 2006b; Dobrica et al. 2009). Thermal effects occurring during the slowing down of the particles in Earth's atmosphere could partly account for these differences. The fact that IOM is ubiquitous in IDPs, AMMs and chondrites precludes its formation in a parent body through hydrothermalism (Flynn et al. 2003). This latter process, in comparison with thermal metamorphism, has generally weak effects on the composition of IOM.

IOM chemical heterogeneity is reflected in the detailed structure of the $3.4-\mu \mathrm{m}$ band (aliphatic hydrocarbons) (Orthous-Daunay et al. 2010b). Therefore, tracking of the $3.4-\mu \mathrm{m}$ band is a potential observational test to infer the presence or absence of IOM in astrophysical contexts. For example in the DISM, infrared spectra point to the presence of an amorphous hydrogenated carbon material, and lack of IOM as observed in chondrites (Dartois et al. 2005). The 3.4- $\mu \mathrm{m}$ band could also help connect chondrite chemical classes to asteroids, still an appreciable challenge, considering the currently achievable signal precision (Licandro et al. 2011).

Finally, we should note that some grains in IDPs and organic meteorites appear to have intact presolar organics that are highly enriched in deuterium, suggesting that at least some cold molecular cloud particles experienced relatively non-violent conditions on arrival in the nebula preserving their original signature, which would be consistent with the "nature" idea. It is especially striking how the highly enriched grains are found adjacent to less enriched grains in meteorites (Duprat et al. 2010; Busemann et al. 2006; Messenger 2002), implying that particles with a wide variety of histories have coalesced, which would probably support a scenario of mixing between "nature" and "nurture". The importance of sample return from various primitive bodies, perhaps not so far represented in the meteorite collections, therefore becomes evident.

\subsection{The nature of asteroids 24 Themis and 65 Cybele}

Rotationally-resolved infrared $(2-4 \mu \mathrm{m})$ spectra of 24 Themis and 65 Cybele indicate that ice and organics are present on the surfaces of these asteroids (Campins et al. 2010; Rivkin \& Emery 2010; Licandro et al. 2011) as indicated by the absorption bands in the range 2.85 to $3.5 \mu \mathrm{m}$ (Fig. 4). A spectrum of the IOM Orgueil (Orthous-Daunay et al. 2010b), highlighting the position of the $3.4 \mu \mathrm{m}$, and one showing the average of six polycyclic aromatic hydrocarbons (Colangeli et al. 1992), are also shown for comparison. These are the first detections of water and organics on asteroid surfaces.

Spectral models for these objects include a mixture of silicates, amorphous carbon and water ice and are shown in Fig. 4 as solid lines. Complex organics are not included in the model: their absence is highlighted by the discrepancy between model and observations. The presence of any surface ice on these asteroids is surprising because of the instability of exposed water ice at their heliocentric distances ( 3.3 AU).

The presence of $\mathrm{H}_{2} \mathrm{O}$ ice and absence of hydrated silicates indicate that 24 Themis and 65 Cybele have not undergone the widespread aqueous alteration or thermal metamorphism recognized in most carbonaceous meteorites. This argues against parent-body processes (such as those described above for SOM in meteorites) as a source of these asteroidal organics. The presence of $\mathrm{C}-\mathrm{H}$ bonds and possibly aromatic groups could be

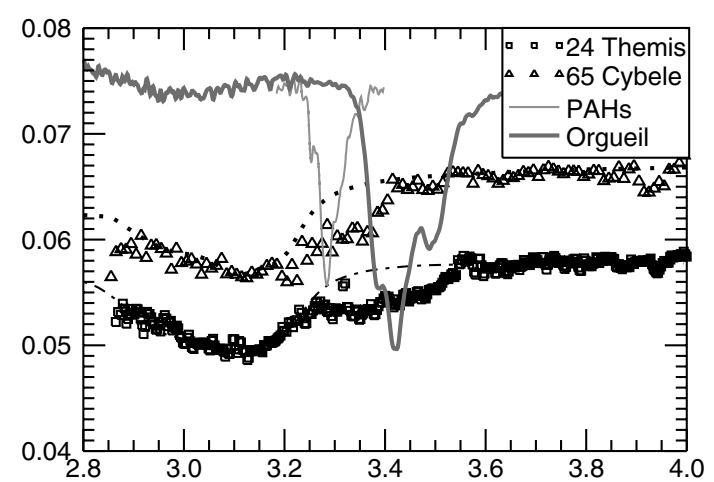

Fig. 4. Reflectance spectra of 65 Cybele and 24 Themis from Licandro et al. (2011) show similarities in the band between 2.85 and $3.5 \mu \mathrm{m}$. The model shown for 24 Themis (Fig. 1, Rivkin \& Emery 2010) and that for 65 Cybele, include water ice, but not organics. A spectrum of the insoluble organic material Orgueil (Orthous-Daunay et al. 2010b) and one showing an average of six polycyclic aromatic hydrocarbons (Colangeli et al. 1992) are included for comparison.

an indication of presolar material ("nature") or irradiation products ("nurture").

\subsection{Organic molecules in cometary components}

Comets are highly relevant to the broad issue of the nature and distribution of complex organic solid material in the solar system. Recent investigations have shown a great compositional variety among comets. The prevalence of solid opaque materials on their surfaces, either as a consolidated crust or as a mantle of loose particles, may distinguish short-period comets from dynamically new comets. The surfaces of comets 9P/Tempel and 103P/Hartley were imaged in 2005 and 2010, respectively, by the Deep Impact spacecraft as part of its original mission and its extended EPOXI mission (see Fig. 5). Both comets appear to have surfaces that are a combination of crusted regions and regions covered with fine particles, having low albedos and reddish colors characteristic of a combination of mafic silicates and carbonaceous materials.

While a number of volatile organic molecules have been identified in the gas phase in cometary comae, of particular interest for the present study is the more refractory solid carbonaceous component of comets. Fomenkova (1999) deduced, from mass spectrometer observations obtained from the comet $\mathrm{P} /$ Halley flyby mission, that $60 \%$ of the carbon on Halley is in the form of complex organic material. This material affects the albedo and color of a comet's nucleus when spatially isolated or mixed with ices. It is of additional interest because this richly organic molecular material can be dispersed in both the inner and outer solar system as a consequence of the dynamical properties of comets. Refractory components of comet dust (organic solids plus minerals) have been collected by aircraft in the stratosphere (a component of interplanetary dust particles, as described above), and by the Stardust spacecraft during its encounter with 81P/Wild 2 (Sandford et al. 2006; Sandford 2008; Clemett et al. 2010).

The organic molecules of cometary origin in the Stardust samples are similar, in many respects, to those found in the matrix material of some carbonaceous chondrite meteorites and IDPs. A detailed analysis of the X-ray absorption spectra of the Stardust particles by Wirick et al. (2009) has shown that these spectra are closer to the spectra of IDPs than those of carbonaceous chondrites, but do not match either category 


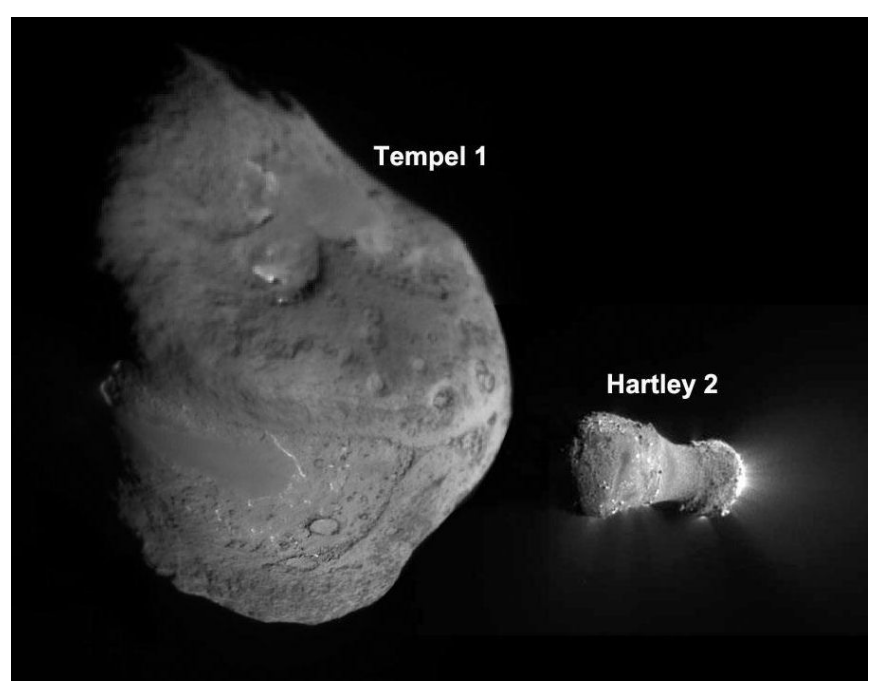

Fig. 5. The surfaces of comets $9 \mathrm{P} / \mathrm{Tempel}$ and $103 \mathrm{P} /$ Hartley imaged, in 2005 and 2010, respectively, by the Deep Impact spacecraft in its original mission and its extended EPOXI mission. P/Tempel is $7.6 \mathrm{~km}$ across and Hartley is $2.2 \mathrm{~km}$ in its long dimension. The figure shows them to the same scale. Image credit: NASA/JPL-Caltech/UMD.

exactly. The Stardust mission particles show extreme heterogeneity (Sandford et al. 2006); some show evidence of a high degree of heating expected from formation in the inner solar system, while others could not have survived such temperatures and must have originated further out. These data corroborate what was previously known from cometary IR spectra showing the existence of crystalline silicates in comets in contrast to their apparent scarcity in the ISM (Ehrenfreund et al. 2004, and references therein). This is also an argument in favor of mixing of "high-temperature" and "low-temperature" materials in the comet-forming region. The "high temperature" group supports the idea of a "nurture" scenario, while the "low-temperature" one could be presolar in origin and promote the "nature" alternative. Aliphatic compounds appear to have withstood temperatures of $\sim 550 \mathrm{C}$, probably because of protection by minerals such as sulfides. Specific identifications include PAHs, with a predominance of single (benzene) and double ring (naphthalene) molecules, but also including much larger PAHs (extending up to $\sim 800$ a.m.u.), as well as their alkylation series resulting from the successive addition of $\mathrm{CH}_{2}$ - subunits. However, it is possible that PAHs formed as a result of the high temperature exposure, which would vouch for "nurture" rather than "nature". A fraction of cometary PAHs containing $\mathrm{N}$ atoms in the form of aromatic nitriles represent a significant difference from meteorite organics (Clemett et al. 2010). Both $\mathrm{O}$ and $\mathrm{N}$ atoms are found in a variety of bonding states, while some particles contain abundant $\mathrm{C}$ atoms that are not in aromatic structures (Sandford 2008). Those particles lacking "aromatization" are instead more likely to support the "nature" situation.

Organic molecules such as $\mathrm{CH}_{4}, \mathrm{C}_{2} \mathrm{H}_{2}, \mathrm{C}_{2} \mathrm{H}_{6}$ and $\mathrm{CH}_{3} \mathrm{OH}$ have been identified in the gas phase of comets and in solid phases from IDPs and Stardust samples. It is therefore reasonable to infer that many short-period comet nuclei, the only ones we have been able to observe so far, have their low albedos and red colors, at least in part, due to the presence of a wide range of complex molecules. Such molecular complexes can occur both in recognizable structures and in more complex aggregates similar to kerogen-like materials in the insoluble organic matter in carbonaceous meteorites. Similarly, the volatile fraction in comet nuclei may include complex molecules analogous to the soluble fraction in the meteorites (e.g., amino and hydroxylic acids, and nitrogen heterocycles). Therefore, comets are plausibly a mixture of native and irradiation products, with their initial composition differing and being a function of their place of formation (Ehrenfreund et al. 2004, and references therein), and advocating for both the "nature" and "nurture" scenarios.

\subsection{The surface composition of Jupiter Trojans}

Jupiter Trojans (JTs) are one of the most intriguing populations of small bodies in the solar system. Currently residing in stable orbits in the Lagrangian points of Jupiter, JTs are believed to have formed at relatively large heliocentric distances, where the solar nebula was rich in frozen volatiles and organics (Dotto et al. 2008; Marzari et al. 2002).

The origin of JTs and how they were trapped in librating orbits around the Jupiter Lagrangian points are still a matter of debate. Several mechanisms have been so far proposed, including capture of fragments of Jovian satellites, trapping of planetesimals orbiting nearby a proto-Jupiter, mass growth of the planet or gas drag capture of short-period comets (Marzari et al. 2002). Morbidelli et al. (2005) suggested that JTs, like Kuiper belt objects (KBOs) and the scattered disk, originated in the planetesimal disk which drove the planetary migration. Therefore, the analysis of the physical properties, in particular the composition of JTs, can help us place important constraints on their origin and early evolution.

Initial compositional interpretations ascribed their low albedos and red, featureless visible spectral slopes at least partly to the presence of organics (Gradie \& Veverka 1980). Comparisons with comets and other outer solar system bodies are not uncommon (e.g., Jewitt 2002), given the similarly dark, spectrally red surfaces, but many low-albedo Main Belt asteroids also exhibit many of the same spectral qualities. Detecting and characterizing the possible organic component of JTs would move us a step closer to understanding the origin and evolutionary processes important for small bodies throughout the solar system.

Statistical analyses of the featureless reflectance spectra have provided some insights. Szabó et al. (2007) reported a correlation between visible colors (from Sloan Digital Sky Survey [SDSS] measurements) and orbital inclination for JTs that manifested itself as an apparent bimodal distribution of spectral slopes. Roig et al. (2008) confirm the SDSS bimodal spectral slope distribution and find a similar distribution in previously published visible spectra. Emery et al. (2011) identify a much stronger bimodality in near-infrared spectral slopes of JTs. This bimodality is present in both the leading and trailing swarms and among the background (non-family member) JTs, and therefore appears to be a signature of the primordial population rather than an artifact of collisional families or capture mechanisms. Spectral similarities between the redder group and some Centaurs and the less-red group and some outer Main Belt asteroids may indicate multiple source regions for JTs.

Of particular interest in the present context are direct searches for organic materials. Strong fundamental vibrational bands of C-H, O-H, and N-H bonds in complex organics occur at wavelengths in the 2.7 to $3.6 \mu \mathrm{m}$ region, and overtones and combinations of these, though significantly weaker, occur at shorter wavelengths in the reflectance spectra of organics. Several authors (e.g. Cruikshank et al. 2001; Emery \& Brown 2003; Dotto et al. 2006; Fornasier et al. 2007; de Luise et al. 2010; Emery et al. 2011; Yang \& Jewitt 2011) have published visible and nearinfrared reflectance spectra of JTs. Despite the expectation that 
JTs formed with abundant volatiles and organics, as for KBOs, they do not appear to exhibit any of the spectral features of these materials on their surfaces. However, as there is more than an order of magnitude difference in the solar flux on Trojans, at 5 AU, compared to the KBOs, the difference in spectral properties of the uppermost surfaces of Trojans and KBOs does not necessarily preclude a similar origin.

Furthermore, the featureless visible spectra seem to rule out a significant quantity of hydrated silicates, crystalline anhydrous silicates, organic materials and ices. Amorphous silicates have been suggested to model the red slopes of JT spectra (Cruikshank et al. 2001; Emery \& Brown 2004), but featureless spectra provide at best a weak constraint on inferred compositions. However, thermal emission spectra of several Trojans show strong spectral emissivity peaks near 10 and $20 \mu \mathrm{m}$, indicating the presence of fine-grained, largely amorphous, anhydrous silicates (Emery et al. 2006; Mueller et al. 2010).

An absorption feature at $\sim 2.3 \mu \mathrm{m}$ found in the spectra of the Centaur Pholus and the TNO $2002 \mathrm{VE}_{95}$ has been attributed to organic materials (Cruikshank et al. 1998; Barucci et al. 2006), and specifically to frozen $\mathrm{CH}_{3} \mathrm{OH}$ or (less likely) hexamethyltetramine. However, near-infrared spectra of $\sim 100$ JTs have been published by various authors, and none of them show this feature. Spectroscopic searches for the strong fundamental absorptions at longer wavelengths $(2.8$ to $4.0 \mu \mathrm{m})$ have been published for eight JTs. No absorption features have been found in these spectra, which strongly limits the type and abundance of organic material on the surfaces of JTs (Cruikshank et al. 2001; Emery \& Brown 2004).

The detection of organic features in the 3.3 to $3.5 \mu \mathrm{m}$ region on two outer Main Belt asteroids, 24 Themis and 65 Cybele, as noted in Sect. 5.2, is instructive. Only one of the JT spectra has high enough $\mathrm{S} / \mathrm{N}$ in this wavelength range to have detected a Themis- or Cybele-like absorption feature. It therefore remains possible that, though not yet detected, organics may be a minor component on the surfaces of JTs. It is likely though, considering their relative proximity to the Sun, that their organic material, if present, could be partially the product of the irradiation of the original surface components. Based on the amount of processing that these surface have undergone the "nurture" scenario would likely be the prevalent one.

\subsection{Polycyclic aromatic and aliphatic hydrocarbons on Saturn's satellite lapetus}

Iapetus, Saturn's third largest satellite, is an icy moon showing spectral signatures of $\mathrm{H}_{2} \mathrm{O}$ ice over more than half its surface. The low-albedo material on Iapetus' surface is concentrated primarily on the leading hemisphere in the sense of the satellite's locked synchronous orbital motion around Saturn, but telescopic studies undertaken since the discovery of the albedo dichotomy by G. D. Cassini in 1671 have failed to convincingly establish the composition of the dark material. New studies, based on data from the Cassini spacecraft demonstrate that there are two low-albedo surface components, one very red in color and the other more nearly neutral (Denk et al. 2010), one native to Iapetus, the other exogenous in nature. The native material is probably ubiquitously present to some degree and was originally evenly distributed on the surface. Both low-albedo components contribute to the dark coloration of the leading hemisphere of Iapetus and are the cause of a temperature enhancement detected in this area of the satellite surface. They are ultimately responsible for the material segregation on the surface of the satellite.
A thermal model of the evolution of Iapetus' surface (Spencer \& Denk 2010), in which $\mathrm{H}_{2} \mathrm{O}$ ice is mobilized by the higher temperatures in the dark hemisphere and re-condensed elsewhere, convincingly reproduces the albedo pattern seen today.

An absorption band at $3.28 \mu \mathrm{m}$, attributed to polycyclic aromatic hydrocarbons, has been identified in the low-albedo material on Iapetus (Cruikshank et al. 2008). The data for that study came from the Visible- Infrared Mapping Spectrometer (VIMS) aboard the Cassini spacecraft that has been operating in orbit around Saturn since late 2004. The detection of PAHs is the first clear identification of a significant chemical component. Current studies are attempting to discern which of the two low-albedo components presents the PAH signature. The characterization of the dark components of Iapetus is important as it sheds light on the origin of the materials and their evolution, as well as the "nature" versus "nurture" question regarding the satellites of Saturn.

\subsection{The surface composition of trans-neptunian objects and Centaurs}

The TNOs or KBOs are small icy bodies (the diameters of known objects range between about 50 and $3000 \mathrm{~km}$ ) that orbit the Sun beyond Neptune. More than 1200 of them have been discovered so far. A number of spectroscopic and dynamical studies (Barucci et al. 2008a, and references therein) have shown that TNOs are remnants of the formation of the solar system that have been subjected to minimal chemical evolution since their formation. Their upper surface layers have however been altered by various space weathering processes (solar and cosmic ion irradiation, collisions) and, for the largest of them, surfaceatmosphere interaction and, possibly, cryovolcanism. A study of their surface compositions can therefore provide clues on solar nebula composition and solar system evolution as well as the origin of their reddening material.

The dynamical evolution of TNOs is believed to have been cataclysmic. A currently popular dynamical model (the Nice model, Levison \& Morbidelli 2003) describes a series of events in the young solar system that caused the migration of the outer giant planets Neptune and Uranus, and the scattering of the planetesimals left over from their formation. TNOs are distinguished (Elliot et al. 2005; Gladman et al. 2008) into several different classes that describe their current locations and orbital characteristics: the classical objects, the resonant objects (such as Pluto, which is in 2:3 orbital resonance with Neptune), the scattered disk objects and the detached objects (like Sedna). In addition, Centaurs (such as Pholus or Chiron) have orbits between those of Jupiter and Neptune. They may be escapees from the Kuiper belt (former TNOs), they are therefore studied in parallel with TNOs, but being closer to us, they are usually brighter.

The chemical history of TNOs is unfortunately not as clearly understood. The concept of "snow lines" in the original nebula, i.e. the distance from the Sun at which materials can condense, provides us with some idea of what should have been the distribution of materials before the neptunian migration. In fact, we can envision that planetesimals, originally formed close to Jupiter, have little more than rock, metal, and water ice as part of their composition. On the other hand, planetesimals originating outward of Saturn would consist of more volatile ices as well as water ice and rock and metal depending on their distance from the Sun and size (Schaller \& Brown 2007). As a result, we expect that the original material distribution would have followed a composition pattern from simple to more complex in traveling from the inner to the outer solar system. Unfortunately this 
simple distribution was apparently altered by giant planet migration and planetesimal scattering like that described in the Nice model, yielding the much more complex picture that we observe today.

Another complication in the unraveling of the chemical history of solar system bodies lies with the processing due to solar and cosmic radiation that has weathered their surfaces, more effectively for those closer to the Sun. The best candidates for our study are probably the smaller TNOs, which are possibly far enough from the Sun to be sufficiently pristine to be useful in our investigation. Larger ones are not as reliable as internal melting and differentiation occurred in their history.

Information about TNO and Centaur surface compositions or physical properties comes from albedo measurements, color measurements, polarimetric observations and spectroscopy. The measured albedos are low (between 0.04 and 0.20), except for a few objects that have albedos of about 0.6 (Pluto, Makemake, Haumea) or even higher ( $\sim 0.86$ for Eris, Stansberry et al. 2008 and $2000 \mathrm{TX}_{300}$; a member of the Haumea family, Elliot et al. 2010). Their generally low albedos, their distances, and their sizes make them very faint and therefore difficult to observe. Even with the largest telescopes available (8 to $10 \mathrm{~m}$ class), only the brightest TNOs can be studied spectroscopically to obtain compositional information. Furthermore, the available TNO spectra extend only to about $2.5 \mu \mathrm{m}$ (Barucci et al. 2008b), a limiting factor when trying to identify surface components whose fundamental spectral features often fall beyond $2.5 \mu \mathrm{m}$.

When spectroscopic data are not available, colors have been obtained for a relatively large sample of objects (around 200). TNOs as well as Centaurs have very diverse colors, from neutral to very red. A taxonomic scheme based on multivariate statistics of the photometric color was proposed by Barucci et al. (2005): four classes were identified indicating different composition and/or evolutional history, with increasingly red colors from the BB (blue objects) to the RR class (reddest objects). The relationship between taxonomical and dynamical classifications has been investigated in detail (Perna et al. 2010) yielding somewhat inconclusive results. No direct correlation between color and dynamical parameters (in particular semi-major axis) was found. However, recent work by Romanishin et al. (2010) shows some evidence for a color gradient with distance from the Sun, if the Inner Classical KBOs (ICKBOs) are considered to be an extension of the Cold Classical population: the Cold Classical objects are predominately red, and the Inner Classical belt objects are a mixture of neutral and red.

When looking at the TNOs, a limited number of high resolution TNO spectra have been modeled using a variety of compositions that include several ices, carbonaceous, and organic materials varying in proportion from object to object. Figure 6 shows the best fitting model of Quaoar, an RR object, orbiting at about 44 AU. Its composition is listed among a few representative cases in Table 1, where the published quantities of the different components have been grouped together into three categories: reddening components (mostly tholins), darkening components (mostly amorphous carbon) and ices (hydrocarbons, $\mathrm{H}_{2} \mathrm{O}, \mathrm{CO}_{2}, \mathrm{CO}, \mathrm{CH}_{3} \mathrm{OH}$ ices). From a compilation of objects with spectra available in the literature (examples shown in Table 1) it appears that composition and semi-major axis $(a)$ also do not correlate: ice abundance does not map with distance and likewise objects with a high abundance of processed dark materials do not orbit closest to the Sun.

Grundy's idea (i.e. the "nature" scenario) of the reddening material being primarily primordial in origin, would imply that the color of the objects vary with the quantity of ice and as a

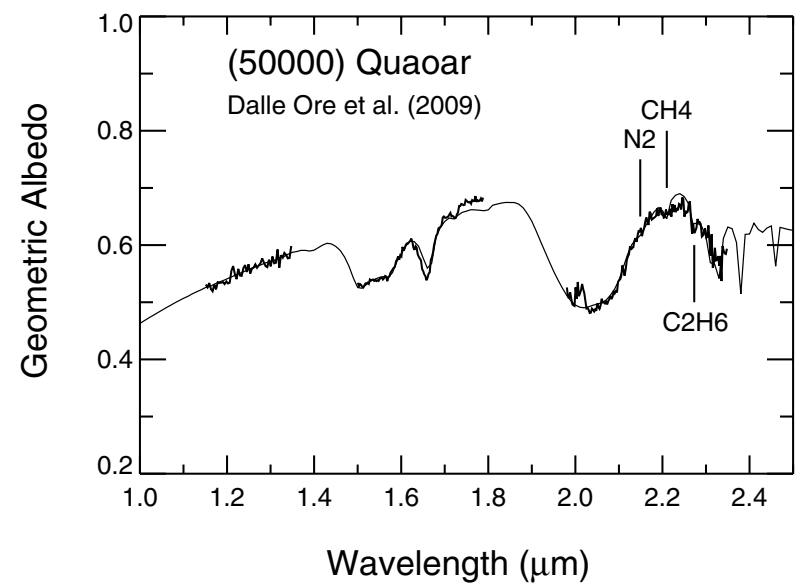

Fig. 6. Quaoar best fitting model (solid thin line) includes about $30 \% \mathrm{H}_{2} \mathrm{O}, 20 \% \mathrm{~N}_{2}$, and $13 \% \mathrm{CH}_{4}$ with traces of $\mathrm{C}_{2} \mathrm{H}_{6}$ ice mixed in with organic materials (Dalle Ore et al. 2009). Hydrocarbon ices features are labeled in the figure. Quaoar is an RR object. The observations were scaled to Stansberry et al. (2008) visual geometric albedo.

Table 1. Selected objects' compositions.

\begin{tabular}{|c|c|c|c|c|c|c|}
\hline Object & $\begin{array}{l}\text { Dyn } \\
\text { class }\end{array}$ & Taxon & $a^{*}$ & $\begin{array}{c}\% \\
\text { reddening }\end{array}$ & $\begin{array}{c}\% \\
\text { darkening }\end{array}$ & $\begin{array}{l}\% \\
\text { ices }\end{array}$ \\
\hline 5145 Pholus $^{a}$ & $\mathrm{C}$ & RR & 20.3 & 34 & 61 & 15 \\
\hline 8405 Asbolus $^{b}$ & $\mathrm{C}$ & $\mathrm{BR}$ & 18.1 & 32 & 59 & 9 \\
\hline $1999 \mathrm{TC}_{36^{c}}{ }^{c}$ & $\mathrm{R}$ & RR & 39.7 & 70 & 22 & 8 \\
\hline 50000 Quaoar $^{d}$ & $\mathrm{C}$ & $\mathrm{RR}$ & 43.6 & 27 & 0 & 73 \\
\hline $90377 \mathrm{~S}$ & D & $\mathrm{RR}$ & 518.6 & 76 & 0 & 24 \\
\hline 90482 Orcus $^{f}$ & $\mathrm{R}$ & BB & 39.2 & 1 & 58 & 40 \\
\hline
\end{tabular}

Notes. ${ }^{(*)}$ Semi-major axis measurements $(a)$ are from JPL Small-Body Database Browser at http://ssd.jpl .nasa.gov/sbdb.cgi

References. ${ }^{(a)}$ Cruikshank et al. (1998); ${ }^{(b)}$ Poulet et al. (2002); ${ }^{(c)}$ Dotto et al. (2003); ${ }^{(d)}$ Dalle Ore et al. (2009); ${ }^{(e)}$ Barucci et al. (2010); (f) Delsanti et al. (2010).

function of distance. However, the measured semi-major axes describe the current position of these objects, after the migration of Neptune had scattered them: it therefore comes as no surprise that their compositions do not relate to their locations.

Out to the Kuiper belt, we expect that current weathering from solar irradiation would affect the surface compositions as a function of distance from the Sun, with the closest objects being most processed. This picture becomes more complicated at distances beyond $50 \mathrm{AU}$ where cosmic rays become predominant (Cooper et al. 2006). As seen in Sect. 5.4 close to the Sun weathering happens on very short timescales, implying that some weathering effects should be seen. In fact, we do see the effects of weathering on the IKBOs, as mentioned above. However, for the TNOs, based on the complete lack of correlation between their composition, and their current semimajor axis, particularly for those that are within the $50 \mathrm{AU}$ radius, two possible explanations become apparent. The first is that not a lot of weathering has happened since Neptune migration repositioned objects to their current locations. The second that the collision rate is comparable to the weathering rate, keeping the surfaces fresh (Melita et al. 2009). In both cases the direct implication is that the weathering that we observe, at least in some of the objects (i.e., the presence of a population of objects with highly processed, dark materials, such as Orcus), must have preceded the migration and must have happened on a short timescale early on. 
Table 2. "Nature" vs. "nurture" summary.

\begin{tabular}{lccc}
\hline \hline Evidence & "Nature" & "Nurture" & $?$ \\
\hline Laboratory studies of refractory carbonaceous materials & $\mathrm{X}$ & $\mathrm{X}$ & - \\
Ion irradiation and colors of C-rich materials & - & $\mathrm{X}$ & - \\
The nature of interstellar organics & $\mathrm{X}$ & - & - \\
Hydrocarbonaceous dust in the ISM & $\mathrm{X}$ & - & - \\
Dense clouds & $\mathrm{X}$ & - & - \\
Evolution of organics from diffuse interstellar clouds to the solar system & $\mathrm{X}$ & - & - \\
Chemistry of protoplanetary disks & - & - & $\mathrm{X}$ \\
Evidence for organics in dusty disks & - & - & $\mathrm{X}$ \\
Refractory organic matter in chondrites and interplanetary dust & $\mathrm{X}$ & $\mathrm{X}$ & - \\
Asteroids 24 Themis \& 65 Cybele & - & - & $\mathrm{X}$ \\
Comets & $\mathrm{X}$ & $\mathrm{X}$ & - \\
The surface composition of Jupiter Trojans & - & - & $\mathrm{X}$ \\
PAHs and aliphatic hydrocarbons on Iapetus & $\mathrm{X}$ & $\mathrm{X}$ & - \\
The surface composition of trans-Neptunian objects and centaurs & $\mathrm{X}$ & $\mathrm{X}$ & - \\
\hline
\end{tabular}

We can therefore conclude, for the TNO case, that the "nature" reddening mechanism, whose traces are apparent in those objects with a lot of ices and red coloration, as well as the "weathering" component visible in the processed materials ("nurture") could be interpreted, for some of the objects, as to date from a time previous to the neptunian migration. At that time both mechanisms might have played a role, but their relative importance is too difficult to trace as their effect on the spectrum of the material is so macroscopically similar as to make it impossible, at this time, to discern the importance of one vs. the other.

\section{Conclusions}

We present an examination of some recent evidence, encompassing a number of laboratory experiments as well as observational data, in an effort to characterize the reddening material common to planetary systems. The available information, discussed at length among the authors, leads us to infer that the red coloration of a variety of objects in the solar system could be due mainly to one of two mechanisms, or more likely to a combination of them: weathering and primordial composition, i.e. "nurture" and "nature".

Laboratory work has given, and is still in the process of giving, us a few tools to aid us in discriminating between the different choices. In this paper we explore in the observations available in the literature the evidence for one process or the other, and view them in the frame of the knowledge acquired through the laboratory studies.

Table 2 summarizes the current status of the "nature" vs. "nurture" debate.

The picture emerging from the various experimental and observational pieces of evidence is a lot more complicated than the simple question of "nature" vs. "nurture" originally posed. There are a few factors playing an important role in the identification of the reddening process:

a) the identification of the reddening material(s) (minerals vs. complex organics);

b) the determination of its origin, i.e. primordial vs. recent;

c) the assessment of its condition with respect to its original state (degree of weathering);

d) the measurement of its contamination with other materials (ices or other).
Some of the key factors outlined above are within reach with the current state-of-the-art telescopes, and observing instruments (e.g. d above), others are far from being solvable.

The identification of the material dwells primarily on the ability to obtain spectroscopic data past the $2.5 \mu \mathrm{m}$ current limit for a number of well chosen objects representing the main taxonomic groups of TNOs, Centaurs, asteroids and Trojans in the hope that the behavior in the near-IR will shed light on their compositions, help map the reddening material(s) throughout the outer solar system, and distinguish minerals from complex organics. This information along with dynamical data might elucidate its origin and weathering level when coupled with laboratory experiments aimed at testing the effects of radiation and other weathering mechanisms in various environments and guide us in the interpretation of the observations.

Nevertheless, materials such as PAHs, indicative of the presence of organics, are difficult to interpret, as their origin could be due to "nurture" as well as "nature". The spectroscopic effects of the "nature" and "nurture" mechanisms on the icy material are practically indistinguishable: while intrinsically different, macroscopically, they both reduce the ratio of $\mathrm{H}$ to $\mathrm{C}$ atoms. Further, with the current state of the art instruments we cannot yet detect the signature features of the organic material, whether primordial or resulting from irradiation, in most of the available observations. A few exceptions include Cassini VIMS data for the Saturn satellites, Triton, Pluto, 24 Themis and 65 Cybele data.

Finally, a model of the dynamical evolution of the outer solar system has to be kept in the picture as the current location of the objects probably does not reflect their original position, therefore complicating the history of the weathering contributions.

In summary, the question at the basis of the "nature" vs. "nurture" debate, i.e. whether ice indeed magnifies the effect of the reddening material, is still to be fully addressed. While some aspects of the problem have been answered or will be in the near future, such as the determination of the amount of ice present in red objects, others, such as the ability to discriminate between reddening agents, are still far from being solvable.

Acknowledgements. This paper is based on the conclusions of the workshop "Organic material in planetary system: "nature" or "nurture"?" held on May 27-29, 2010 at the Centre International d'Ateliers Scientifiques de l'Observatoire de Paris with the participation of all the authors. C.M.D.O. aknowledges support from the Centre International d'Ateliers Scientifiques de l'Observatoire de Paris. N.P.A. acknowledges support from the NASA postdoctoral program at Ames Research Center administered by Oak Ridge Associated Universities through a contract with NASA. 


\section{References}

O’D. Alexander, C. M., Russell, S. S., Arden, J. W., et al. 1998, M\&PS, 33, 603 Alexander, C. M. O’D., Fogel, M., Yabuta, H., \& Cody, G. D. 2007, GeCoA, 71, 4380

Apai, D., Connolly, H. C., Jr., \& Lauretta, D. S. 2010, Protoplanetary Dust: Astrophysical and Cosmochemical Perspectives, 230

Baratta, G. A., Brunetto, R., Leto, G., et al. 2008, JRSp, 39, 211

Barucci, A., Belskaya, I. N. Fulchignoni, M., \& Birlan, M. 2005, AJ, 130, 1291

Barucci, M. A., Merlin, F., Dotto, E., et al. 2006, A\&A, 455, 725

Barucci, M. A., Boehnhardt, H., Cruikshank, D. P., et al. 2008a, The Solar System Beyond Neptune (Tucson: U. Arizona Press), 3

Barucci, M. A., Brown, M. E., Emery, J. P., et al. 2008b, The Solar System Beyond Neptune (Tucson: U. Arizona Press), 143

Barucci, M. A., Morea Dalle Ore, C., Alvarez-Candal, A., et al. 2010, AJ, 140, 2095

Benecchi, S. D., Noll, K. S., Grundy, W. M., et al. 2009, Icarus, 200, 292

Bernard, J.-M., Quirico, E., Brissaud, O., et al. 2006, Icarus, 185, 301

Bonal, L., Quirico, E., Bourot-Denise, M., \& Montagnac, G. 2006a, GeCoA, 70, 1849

Bonal, L., Quirico, E., Montagnac, G. \& Reynard, B. 2006b, LPI, 37, 2271

Bonal, L., Bourot-Denise, M., Quirico, E., et al. 2007, GeCoA, 71, 1605

Brunetto, R., Barucci, M. A., Dotto, E., \& Strazzulla, G. 2006, ApJ, 644, 646

Busemann, H., Young, A. F., Alexander, C. M. O’D., et al. 2006, Science, 312, 727

Busemann, H., Alexander, C. M. O’D., \& Nittler, L. R. 2007, M\&PS, 42, 1387

Campins, H., Hargrove, K., \& Pinilla-Alonso, N. 2010, Nature, 464, 1320

Carr, J. S., \& Najita, J. R. 2008, Science, 319, 1504

Chiar, J. E., \& Pendleton, Y. J. 2008, in Organic Matter in Space, ed. S. Kwok, \& S. A. Sanford (Cambridge: Cambridge Univ. Press), IAU Symp., 251, 35

Chiar, J. E., Pendleton, Y. J., Allamandola, L., et al. 2011, ApJ, submitted

Clark, R. N., Brown, R. H., Jaumann, R., et al. 2008, Icarus, 193, 372

Clemett, S. J., Sandford, S. A., Nakamura-Messenger, K., et al. 2010, M\&PS, 45,701

Cody, G. D., \& Alexander, C. M. O’D. 2005, GeCoA, 69, 1085

Colangeli, L., Mennella, V., Baratta, G. A., et al. 1992, ApJ, 396, 369

Cooper, J. F., Hill, M. E., Richardson, J. D., et al. 2006, Physics of the Inner Heliosheath, 858, 372

Cruikshank, D. P., Roush, T. L., Bartholomew, M. J., et al. 1998, Icarus, 135, 389

Cruikshank, D. P., Dalle Ore, C. M., Roush, T. L., et al. 2001, Icarus, 153, 348

Cruikshank, D. P., Imanaka, H., \& Dalle Ore, C. M. 2005, Adv. Space Res., 36, 178

Cruikshank, D. P., Wegryn, E., Dalle Ore, C. M., et al. 2008, Icarus, 193, 334

Cuzzi, J., Clark, R., Filacchione, G., et al. 2009, Saturn from Cassini-Huygens (Springer), 459

Dalle Ore, C. M., Barucci, M. A., Emery, J. P., et al. 2009, A\&A, 501, 349

Dartois, E., \& Muñoz-Caro, G. M. 2007, A\&A, 476, 1235

Dartois, E., Muñoz-Caro, G. M., Deboffle, D., \& d'Hendecourt, L. 2004, A\&A, 423, L33

Dartois, E., Muñoz Caro, G. M., Deboffle, D., et al. 2005, A\&A, 432, 895

Dartois, E., Geballe, T. R., Pino, T., et al. 2007, A\&A, 463, 635

Debes, J. H., Weinberger, A. J., \& Schneider, G. 2008, ApJ, 673, L191

Delsanti, A., Merlin, F., Guilbert-Lepoutre, A., et al. 2010, A\&A, 520, A40

de Luise, F., Dotto, E., Fornasier, S., et al. 2010, Icarus, 209, 586

Denk, T., Neukum, G., Roatsch, T., et al. 2010, Science, 327, 435

Dobrica, E., Engrand, C., Quirico, E., et al. 2009, LPI, 40, 1688

Doressoundiram, A., Boehnhardt, H., Tegler, S. C., \& Trujillo, C. 2008, The Solar System Beyond Neptune, 91

Dotto, E., Barucci, M. A., Boehnhardt, H., et al. 2003, Icarus, 162, 408

Dotto, E., Fornasier, S., Barucci, M. A., et al. 2006, Icarus, 183, 420

Dotto, E., Emery, J. P., Barucci, M. A., et al. 2008, The Solar System Beyond

Neptune (Tucson: University of Arizona Press), 383

Duley, W. W. 2000, ApJ, 528, 841

Duley, W. W., Jones, A. P., \& Williams, D. A. 1989, MNRAS, 236, 709

Duprat, J., Dobrica, E., Engrand, C., et al. 2010, Science, 328, 742

Ehrenfreund, P., Charnley, S. B., \& Wooden, D. 2004, Comets II, 115

Elliot, J. L., Kern, S. D., Clancy, K. B., et al. 2005, AJ, 129, 1117

Elliot, J. L., Person, M. J., Zuluaga, C. A., et al. 2010, Nature, 465, 897

Emery, J. P., \& Brown, R. H. 2003, Icarus, 164, 104

Emery, J. P., \& Brown, R. H. 2004, Icarus, 170, 131

Emery, J. P., Cruikshank, D. P., \& Van Cleve, J. 2006, Icarus, 182, 496

Emery, J. P., Burr, D. M., \& Cruikshank, D. P. 2011, AJ, 141, 25

Fedele, D., Pascucci, I., Brittain, S., et al. 2011, ApJ, 732, 106

Feigelson, E. D., \& Montmerle, T. 1999, ARA\&A, 37, 363

Flynn, G. J., Keller, L. P., Feser, M., Wirick, S., \& Jacobsen, C. 2003, GeCoA, 67, 4791

Fomenkova, M. N. 1999, Space Sci. Rev., 90, 109

Fornasier, S., Dotto, E., Hainaut, O., et al. 2007, Icarus, 190, 622
Gladman, B., Marsden, B. G., \& Vanlaerhoven, C. 2008, The Solar System Beyond Neptune (Tucson: University of Arizona Press), 43

Gradie, J., \& Veverka, J. 1980. Nature, 283, 840

Grigorieva, A., Thébault, P., Artymowicz, P., \& Brandeker, A. 2007, A\&A, 475, 755

Grundy, W. M. 2009, Icarus, 199, 560

Grundy, W. M., \& Stansberry, J. A. 2003, EM\&P, 92, 331

Güdel, M., Lahuis, F., Briggs, K. R., et al. 2010, A\&A, 519, 113

Hoffmann, M., Fink, U., Grundy, W. M., \& Hicks, M. 1993, JGR, 98, 7403

Horner, J., \& Lykawka, P. S. 2010, Internat. J. Astrobiol., 9, 227

Imanaka, H., Khare, B. N., Elsila, J. E., et al. 2004, Icarus, 168, 344

Jäger, C., Krasnokutski, S., Staicu, A., et al. 2006, ApJS, 166, 557

Jewitt, D. 2002, AJ, 123, 1039

Jones, A. P. 1990, MNRAS, 247, 305

Jones, A. P. 2005, IAU Symp., 231, 34

Jones, A. P., \& Nuth III, J. A. 2011, A\&A, 530, A44

Jones, A. P., Duley, W. W., \& Wiliams, D. A. 1990, QJRAS, 31, 567

Jones, A. P., Tielens, A. G. G. M., \& Hollenbach, D. J. 1996, ApJ, 469, 740

Kanuchova, Z., Baratta, G. A., Garozzo, M., et al. 2010, A\&A, 517, A60

Köhler, M., Mann, I., \& Li, A. 2008, ApJ, 686, L95

Lamy, P., \& Toth, I. 2009, Icarus, 201, 674

Licandro, J., Campins, H., Kelley, M., et al. 2011, A\&A, 525, A34

Marzari, F., Scholl, H., Murray, C., \& Lagerkvist, C. 2002, In Asteroids III (Tucson: Univ. Arizona Press), 725

Melita, M. D., Strazzulla, G., \& Bar-Nun, A. 2009, Icarus, 203, 134

Mennella, A., Bersanelli, M., Butler, R. C., et al. 1999, ApJ, 524, L71

Mennella, V. 2008a, ApJ, 682, L101

Mennella, V. 2008b, IAU Symp., 251, 459

Mennella, V. 2010, ApJ, 718, 867

Mennella, V., Brucato, J. R., Colangeli, L., \& Palumbo, P. 2002, ApJ, 569, 531

Mennella, V., Baratta, G. A., Esposito, A., et al. 2003, ApJ, 587, 727

Messenger, S. 2002. P\&SS, 50, 1221

Micelotta, E. R., Jones, A. P., \& Tielens, A. G. G. M. 2010a, A\&A, 510, A37

Micelotta, E. R., Jones, A. P., \& Tielens, A. G. G. M. 2010b, A\&A, 510, A36

Micelotta, E. R., Jones, A. P., \& Tielens, A. G. G. M. 2011, A\&A, 526, A52

Morbidelli, A., Levison, H. F., Tsiganis, K., \& Gomes, R. 2005, Nature, 435, 462

Moroz, L., Baratta, G., Strazzulla, G., et al. 2004, Icarus, 170, 214

Muñoz Caro, G. M., Matrajt, G., Dartois, E., et al. 2006, A\&A, 459, 147

Mueller, M., Marchis, F., Emery, J. P., et al. 2010, Icarus, 205, 505

Najita, J. R., Carr, J. S., Strom, S. E., et al. 2010, ApJ, 712, 274

Nakamura-Messenger, K., Messenger, S., Keller, L. P., et al. 2006, Science, 314, 1439

Öberg, K. I., Qi, Ch., Fogel, J. K. J., et al. 2010, ApJ, 720, 480

Oro, J. 1961, Nature, 191, 1193

Orthous-Daunay, F.-R., Quirico, E., Lemelle, L., et al. 2010a, E\&PSL, 300, 321

Orthous-Daunay, F.-R., Quirico, E., Beck, P., et al. 2010b, LPI, 1533, 1803

Owen, T. C., Cruikshank, D. P., Dalle Ore, C. M., et al. 2001, Icarus, 149, 160

Pascucci, I., \& Sterzik, M. 2009, ApJ, 702, 724

Pascucci, I., Gorti, U., Hollenbach, D., et al. 2006, ApJ, 651, 1177

Pascucci, I., Hollenbach, D., Najita, J., et al. 2007, ApJ, 663, 383

Pascucci, I., Apai, D., Luhman, K., et al. 2009, ApJ, 696, 143

Pendleton, Y. J., \& Allamandola, L. J. 2002, ApJS, 138, 75

Pendleton, Y. J., Sandford, S. A., Allamandola, L. J., et al. 1994, ApJ, 437, 683

Perna, D., Barucci, M. A., Fornasier, S., et al. 2010, A\&A, 510, A53

Petrie, S., Stranger, R., \& Duley, W. W. 2003, ApJ, 594, 869

Pety, J., Teyssier, D., Foss'e, D., et al. 2005, A\&A, 435, 885

Pino, T., Dartois, E., Cao, A.-T., et al. 2008, A\&A, 490, 665

Poulet, F., Cuzzi, J. N., Cruikshank, D. P., et al. 2002, Icarus, 160, 313

Pontoppidan, K. M., Salyk, C., Blake, G. A., et al. 2010, ApJ, 720, 887

Quirico, E., Raynal, P. I., \& Bourot-Denise, M. 2003, M\&PS, 38, 795

Quirico, E., Borg, J., Raynal, P.-I., et al. 2005, Planet. Space Sci., 53, 1443

Quirico, E., Montagnac, G., Rouzaud, J. N., et al. 2009, E\&PSL, 287, 185

Remusat, L., Derenne, S., Robert, F., \& Knicker, H. 2005, GeCoA, 69, 3919

Rivkin, A. S., \& Emery, J. P. 2010, Nature, 464, 1322

Roig, F., Ribeiro, A. O., \& Gil-Hutton, R. 2008, A\&A, 483, 911

Romanishin, W., Tegler, S. C., \& Consolmagno, G. J. 2010, AJ, 140, 29

Sandford, S. A. 2008, Ann. Rev. Anal. Chem., 1, 549

Sandford, S. A., Allamandola, L. J., Tielens, A. G. G. M., et al. 1991, ApJ, 371, 607

Sandford, S. A., Aléon, J., Alexander, C. M. O.'D., et al. 2006, Science, 314, 1720

Serra Díaz-Cano, L., \& Jones, A. P. 2008, A\&A, 492, 127

Scott, A., \& Duley, W. W. 1996, ApJ, 472, L123

Schaller, E. L., \& Brown, M. E. 2007, ApJ, 659, L61

Schneider, G., Weinberger, A. J., Becklin, E. E., et al. 2009, AJ, 137, 53

Sellgren, K., Smith, R. G., \& Brooke, T. Y. 1994, ApJ, 433, 179

Smith, R. G., Sellgren, K., \& Brooke, T. Y. 1993, MNRAS, 263, 749

Spencer, J., \& Denk, T. 2010, Science, 327, 432 
A\&A 533, A98 (2011)

Stansberry, J., Grundy, W., Brown, M., et al. 2008, The Solar System Beyond Neptune (Tucson: U. Arizona Press), 161

Strazzulla, G., \& Baratta, G. A. 1991, A\&A, 241, 310

Szabó, G., Ivezić, Ž., Jurić, M., \& Lupton, R. 2007, MNRAS, 377, 1397

Thompson, W. R., Murray, B. G. J. P. T., Khare, B. N., \& Sagan, C. 1987, J. Geophys. Res., 92, 14933
Volk, K., \& Malhotra, R. 2008, ApJ, 687, 714

Wirick, S., Flynn, G. J., Keller, L. P., et al. 2009, M\&PS, 44, 1611

Yabuta, H., Naraoka, H., Sakanishi, K., \& Kawashima, H. 2005, M\&PS, 40, 779

Yabuta, H., Alexander, C. M. O.'D., Fogel, M. L., et al. 2010. M\&PS, 45, 1446 Yang, B., \& Jewitt, D. 2011, AJ, 141, 95 